\title{
Zur Morphogenese des Billetales zwischen Witzhave und Bergedorf (bei Hamburg)
}

\author{
Antje Reclam *)
}

Landform evolution, glacial morphology, moraine, glacial valley, overridden, Middle Pleistocene (Saalian), borehole section, langitudinal profile, terrace, sand, gravel, granulometry.

Northwestern German Plain (Bille valley, Witzhave/Bergedorf), Schleswig-Holstein. TK 2427, 2527

Kurzfassung: Mit Hilfe sedimentpetrographischer und gefügekundlicher Methoden wurden neue Untersuchungen zur Genese des Billetales (östlich von Hamburg) durchgeführt. An den untersuchten Stellen zieht sich die Niendorfer Moräne (Mittlere Saale-Vereisung) in das Tal hinab. Eine Urform des Billetales ist damit möglicherweise bereits während der Saale-Eiszeit gebildet worden. Durch den Fuhlsbüttler Vorstoß (Spätsaale) wurde das Gebiet erneut vom Eis überfahren. Erst in der Weichsel-Eiszeit kam es zur Ausbildung der heutigen Talform. Im meist etwa $700 \mathrm{~m}$ breiten Billetal lassen sich zwei Terrassenniveaus deutlich unterscheiden. Die ca. $1 \mathrm{~m}$ mächtigen Terrassensedimente bestehen aus groben Sanden und Kiesen. In diese Sedimente hat sich gegen Ende der Weichsel-Eiszeit die Bille eingeschnitten. Die etwa $200 \mathrm{~m}$ breite Talaue wird heute nur noch bei Schneeschmelze in ganzer Breite vom Wasser durchflossen. Hinweise darauf, daß das Weichsel-Eis im Billetal bis an die Elbe vorgestoßen sei, fanden sich nicht.

\section{[On the Morphogenesis of the Bille Valley between Witzhave and Bergedorf (near Hamburg)]}

A b s r a c t : New investigations into the genesis of the Bille valley (east of Hamburg) were conducted by means of sediment-petrographical and structural analyses. In the investigated profiles the Niendorf till (Middle Saalian) was found to reach downslope into the valley. Thus an early form of the Bille valley may have existed as early as the Saalian and was overridden again by the late Saalian Fuhlsbüttel advance. The main forming process, however, took place during the Weichselian. The Bille valley today has a width of approximately $700 \mathrm{~m}$. Two terraces can be clearly distinguished. The terrace sediments are about $1 \mathrm{~m}$ thick and consist mainly of coarse sand and gravel. Into these sediments the river Bille cut a $200 \mathrm{~m}$ wide inner valley at the end of the Weichselian. Only during the snow-melting in spring the complete inner valley is inundated today. No hints can be found to a Weichselian ice-advance along the Bille valley towards the Elbe river.

\section{Einleitung}

Das Billetal war bisher nur selten Gegenstand geowissenschaftlicher Untersuchungen.

- Am ausführlichsten befaßt sich Pfefferle (1935) in seiner Arbeit über die Geestrandtäler nördlich und südlich der Elbe mit dem Billetal. Er kommt zu dem Schluß, daß sich das Billetal als Schmelzwassertal der Weichselvereisung gebildet habe, während deren Verlauf es auf Grund des Klimawechsels zur Akkumulation einer Talsandterrasse gekommen sei.

- Illies (1952) vermutet dagegen, daß die Au, die bei Aumühle in die Bille mündet, älter sei als die Bille selbst. Das Autal sei bereits während der Saale-Eiszeit als Schmelzwasserabfluß entstanden.

- JANZA (1961) schließt sich dieser Deutung weitgehend an. Durch rückwärtigen Schmelzwasserabfluß des tauenden Warthe-Eises sei zunächst die Sachsenwaldau entstanden, die anfangs über Schönningstedt zur Glinder Au abfloß und erst später einen weiter südlichen Lauf nahm und bei Bergedorf in die Urelbe mündete.

*) Anschrift der Verfasserin: Dipl.-Geographin A n t je R e cla m, Geol. Landesamt Hamburg, Oberstraße 88, 2000 Hamburg 13. 
- GRIPP (1964) bezeichnet die vom Schmelzwasser in saalezeitliche Ablagerungen eingeschnittene schmale, tiefe Rinne der Bille als „Rinnensander", der diese spezielle Form dadurch erhalten habe, daß das Gletschertor bei Witzhave nur wenige Kilometer vom Urstromtal der Elbe entfernt lag. Durch den Höhenunterschied (von NN $+23 \mathrm{~m}$ bei Witzhave auf das damals bei etwa NN $-25 \mathrm{~m}$ liegende Elbtal) fanden die Schmelzwässer ein ungleich stärkeres Gefälle vor als im Bereich der flach geschütteten Kegelsander (z. B. bei Neumünster), die erheblich weiter von der Erosionsbasis entfernt lagen.

Während sich die genannten Bearbeiter darüber einig waren, daß die Entstehung des Billetales unter subaerischen Bedingungen stattgefunden habe, glaubt JASCHKE (1976) in Anlehnung an Olbricht (1909) und Stoller (1914), daß das Eis der Weichsel-Vereisung die Elbe im Raum Lauenburg-Lüneburg überschritten habe. Die Entstehung des Billetales habe sich dabei in vier Phasen vollzogen:

1. Während des Hochglazials der Weichsel-Vereisung, als das Inlandeis sich aus dem Raum Lüneburg zurückzog, blieb im Bereich des heutigen Billetales ein flacher Eislobus liegen, der im NE mit dem sich bei Witzhave regenerierenden Eisrand in Verbindung stand. Subglazial abfließende Schmelzwässer räumten zu dieser Zeit das „Tunneltal“ der Bille sowie die Dallbekschlucht und andere Kerbtäler am Rand des Elbe-Urstromtales aus. Über der Abtragungssohle wurden im Billetal etwa 1-1,5 m mächtige schräggeschichtete Fein- bis Mittelsande mit einzelnen Geröllen abgelagert.

2. Während des späten Hochglazials taute der Bille-Lobus ab und hinterließ eine etwa 1,5-2 m mächtige Moräne. Sie besteht aus einer Matrix von Sand, Schluff und Geschieben von bis zu 1,5 m Durchmesser, in die vereinzelt Linsen geschichteter Sande eingeschaltet sind. Die Moräne wird von geringmächtigen Nachschüttsanden bedeckt.

3. Während des Spätglazials, als die Umgebung des Billetales bereits flächendeckende Vegetation trug, schnitten sich die Schmelzwässer des jetzt weiter im $\mathrm{N}$ liegenden Eisrandes bis in das Niveau der liegenden Vorschüttsande des Bille-Lobus ein. Dabei entstand ein System von Erosionsterrassen, die im wesentlichen aus dem Material der zwischen die Schmelzwassersande eingeschalteten Moräne aufgebaut sind.

4. Im Postglazial stellte sich zunächst ein Gleichgewicht zwischen Abtragung und Ablagerung ein. Erst mit der Rodung der natürlichen Wälder begann wieder eine Akkumulation feiner Bodenbestandteile im Bereich der Talaue, die bis heute anhält.

Das Ziel meiner Arbeit war es, durch stärker geländeorientierte Untersuchungen die Deutung der Morphogenese des Billetales zu überprüfen. Sedimentpetrographische Untersuchungen, Gefügemessungen, Luftbildauswertung und Auswertung der Unterlagen des Bohrarchivs des Geologischen Landesamtes Hamburg sollten dazu beitragen, die Gegensätze zu klären und zu gesicherten Aussagen über die Entstehung des Billetales zu kommen. Die Lage des Untersuchungsgebietes ergibt sich aus Abb. 1.

\section{Die heutige Bille}

Die rezente Bille ist ein kleiner, auch innerhalb der großen Talmäander stark mäandrierender Fluß, der südlich Witzhave etwa $3 \mathrm{~m}$ breit und $20-30 \mathrm{~cm}$ tief ist (Abb. 2). Bis Reinbek nimmt die Bille an Breite und Tiefe leicht $\mathrm{zu}$.

Der Oberlauf ist wenig anthropogen beeinflußt, während in Reinbek schon früh zur Betreibung einer Mühle ein Teich aufgestaut wurde (vgl. Erstausgabe des Meßtischblattes 2427 Glinde von 1880). Die Gegend um die Mündung in die Elbe ist bereits seit dem 15. Jh. (UPHOFF 1949) von Menschenhand verändert worden. 


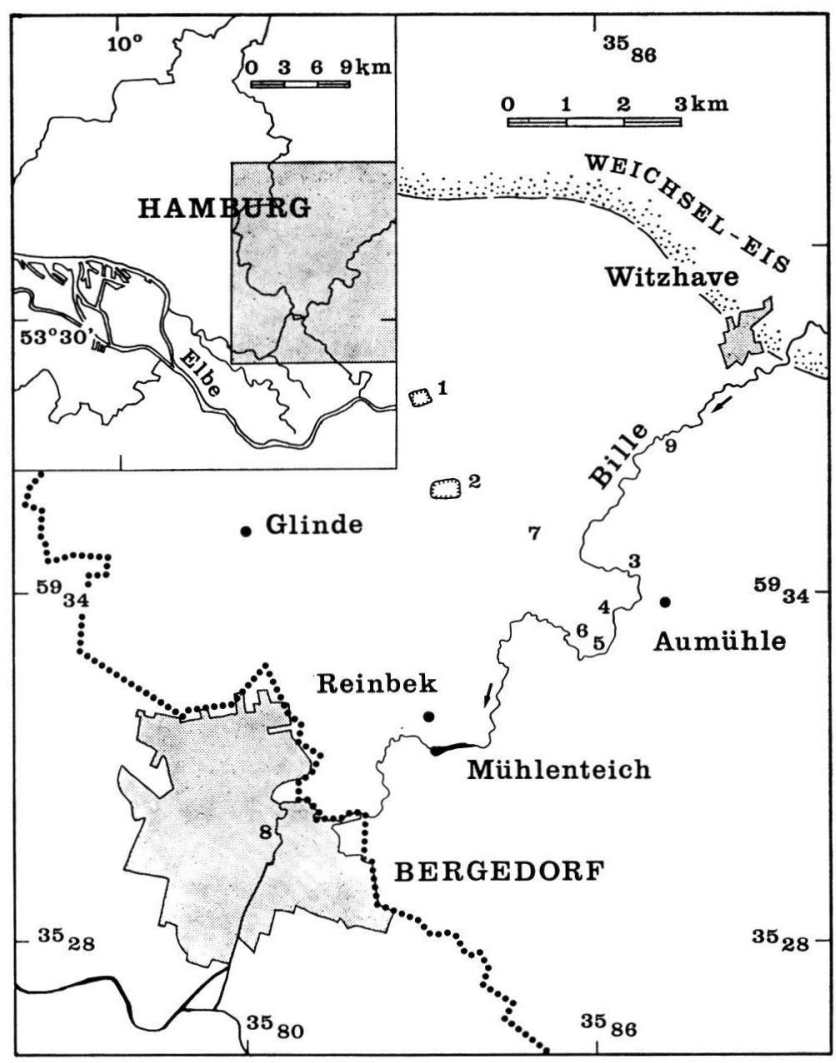

Abb. 1: Ubersichtskarte: 1 = Sandgrube Neu-Schönningstedt; 2 = Kalksandsteinwerk Neu-Schönningstedt; $3=$ Prallufer der Bille im Sachsenwald; $4=$ Unterführung Krabbenkamp; 5 u. $6=$ Baugruben Krabbenkamp; $7=$ Aufschluß Klingeberg; $8=$ Bohrung Billebogen; $9=$ Doktorbrücke.

Die natürlichen Veränderungen gehen unter dem heutigen Klima nur sehr langsam voran.

An drei Stellen im Oberlauf wurden Sedimentproben aus der Bille entnommen und im Labor untersucht. Zwei der Proben entsprechen mit 99,7\% und 99,5\% Feinerdeanteil (im pedologischen Sinne) den typischen Ablagerungen eines heutigen Flusses von der Größenordnung der Bille; sie transportiert fast ausschließlich Mittel- und Grobsand, der in rollendem Transport an der Sohle des Flusses entlangbewegt wird. Die Gewässersohle ist an den meisten Stellen von wandernden Rippeln und Sandbänken bedeckt. Probe a (Abb. 3) wurde an einer Stelle mit rezenter Tiefenerosion entnommen, im Flußbett befinden sich hier grober Kies und Steine. Dementsprechend zeigt auch die Probe eine auffallend andere Korngrößenverteilung: sie enthält viel gröberes Material, der Kiesanteil beträgt fast $20 \%$. Das gröbere Material stammt aus dem Untergrund und ist als Restsediment einer aufgearbeiteten Moräne anzusehen.

\section{Die Morphologie des Billetales}

Bei der Frage nach der Genese des Billetales ist zunächst die Frage zu beantworten, ob es sich um ein subaerisch oder subglazial gebildetes Tal handelt. Subaerische Täler 


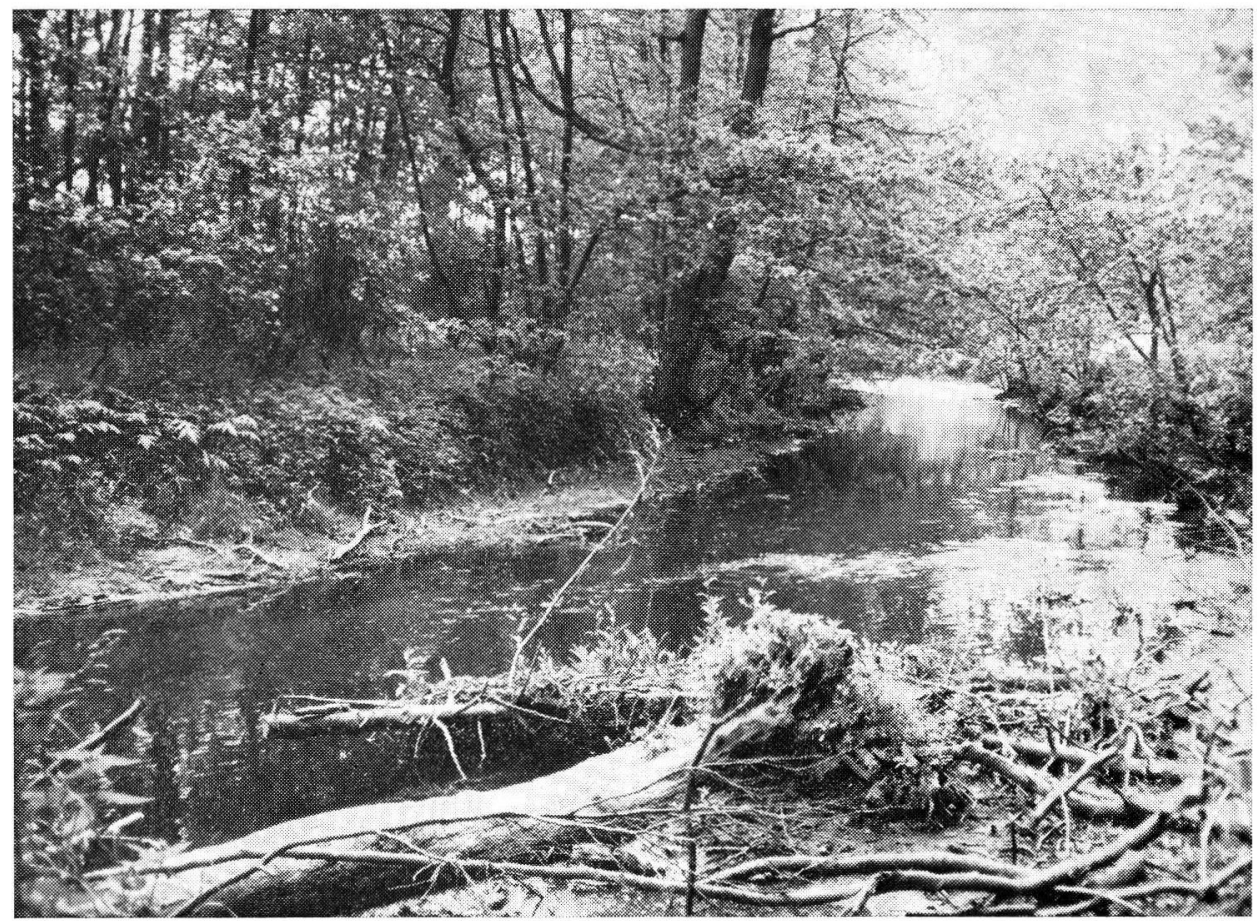

Abb. 2: Die heutige Bille nördlich von Aumühle. Blick nach NW; R 35.864 H 59.345.

unterscheiden sich in Gefälle, Querschnitt und in der Ausbildung der Nebentälchen erheblich von subglazial gebildeten Tunneltälern (GRUBE 1968). Tunneltäler werden durch eine unregelmäßige Talform, also stark wechselnde Breite und weitgehend aufgelöste Talflanken ebenso charakterisiert wie durch Übertiefungen in Fließrichtung, GrundmoränenQuerrippen und Drumlins auf dem Talboden. Außerdem sind keine ausgeprägten Prallund Gleithänge zu finden, keine Terrassen, und die Nebentäler münden oft mit deutlicher Gefällstufe. Insgesamt haben subglazial entstandene Täler eine sehr unruhige Morphologie (GRIPP 1964). An den Talflanken sollte Grundmoräne des Eisvorstoßes nachzuweisen sein, der das Tal geschaffen hat. So hat z. B. Hомсі (1974) weichselzeitliche Grundmoräne an den Flanken des Stellmoorer Tunneltales nachgewiesen.

Das Billetal zeigt keines dieser Merkmale. Die Talbreite des weichselzeitlichen Billetales (Abb. 4) liegt im untersuchten Gebiet zwischen Witzhave und Reinbek bei ungefähr 700 Metern; die einzige Ausnahme findet sich im Bereich von Sachsenwaldau, wo sich das Tal auf $250 \mathrm{~m}$ verengt. Übertiefungen in Fließrichtung, Grundmoränen-Querriegel und Drumlins auf dem Talboden konnten an keiner Stelle gefunden werden. Dafür treten besonders deutlich Prall- und Gleithänge auf, und von Witzhave bis Reinbek lassen sich an beiden Ufern mehrere Terrassenniveaus nachweisen (Abb. 3, 4). Die heutigen Trockentäler münden ohne Gefällstufe auf die obere Terrasse, während die Täler, in denen heute noch Wasser fließt, sich bis zur Talaue eingetieft haben.

Die Morphologie des Billetales mit den großen Talmäandern (siehe Abb. 4) entspricht also in keiner Weise dem Erscheinungsbild eines Tunneltales. 


\section{Die Terrassen im Billetal}

Talterrassen sind als ehemalige Talböden Indizien für früher abgelaufene morphogenetische Vorgänge und somit wichtige morphologische Zeugen der Entstehungsgeschichte eines Tales. Die horizontale Ausdehnung der Terrassen hängt ab von der Wasserführung des Flusses, der Dauer der Lateralerosion und den Gesteinsunterschieden.

Eine durch Geländebeobachtung, Luftbildauswertung und Vergleich mit topographischen Karten ausgeführte Kartierung der Terrassen im Billetal (Abb. 4) und deren Uber-

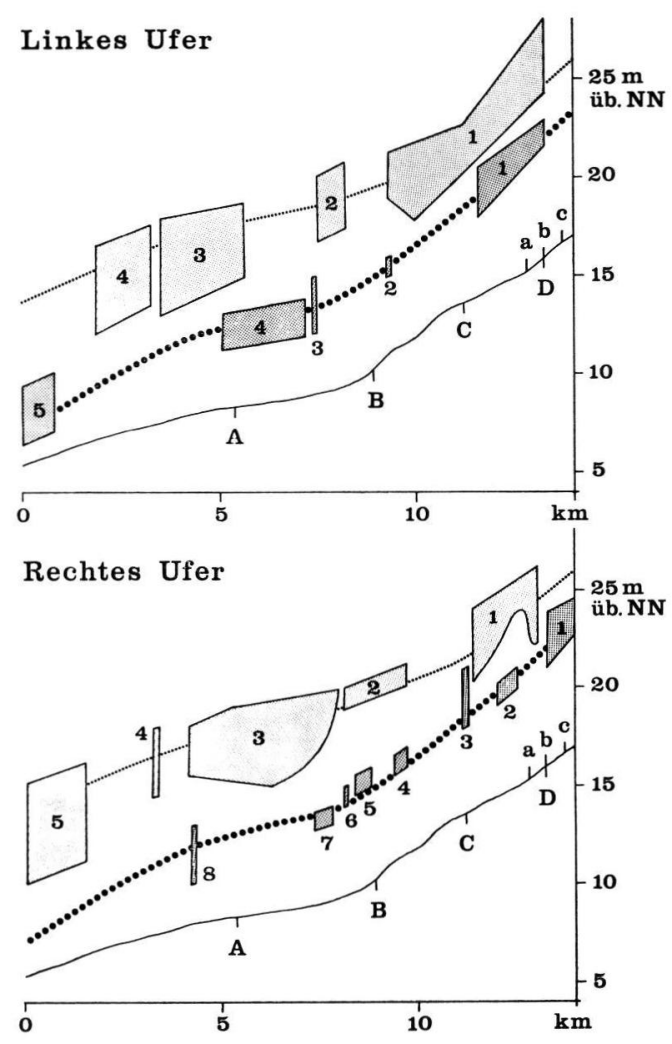

Abb. 3: Schematische Längsprofile der Bille mit Terrassen. Zur Lage der Terrassen siehe Abb. 4. $\mathrm{A}=$ S-Bahnbrücke bei Blockstelle Silk; $\mathrm{B}=$ Mündung der Au; C = Brücke Sachsenwaldau; $\mathrm{D}=$ Doktorbrücke; $\mathrm{a}=$ Probe $500 \mathrm{~m}$ flußabwärts der Doktorbrücke; $\mathrm{b}=$ Probe Doktorbrücke; $\mathrm{c}=$ Probe $500 \mathrm{~m}$ flußaufwärts der Doktorbrücke.

tragung ins Längsprofil des Flusses (Abb. 3) macht deutlich, daß mehrere übereinanderliegende Terrassenniveaus vorhanden sind. Viele davon dürften jedoch als Lokalterrassen nicht zur Rekonstruktion der Talgeschichte heranzuziehen sein.

GRIPP (1933) hat am Krabbenkamp vier übereinanderliegende Terrassenstufen beschrieben. Die obere Terrasse, die sich von der unteren, durchgehend verfolgbaren Terrasse klar abhebt, läßt dort tatsächlich mehrere Verebnungen und Stufen in ihrer zur Talaue leicht geneigten Oberfläche erkennen (Abb. 5, Profil 1). Auch fällt auf, daß die Höhenlage der oberen Terrasse viel stärker variiert als die der unteren (Abb. 3). Es sollte 


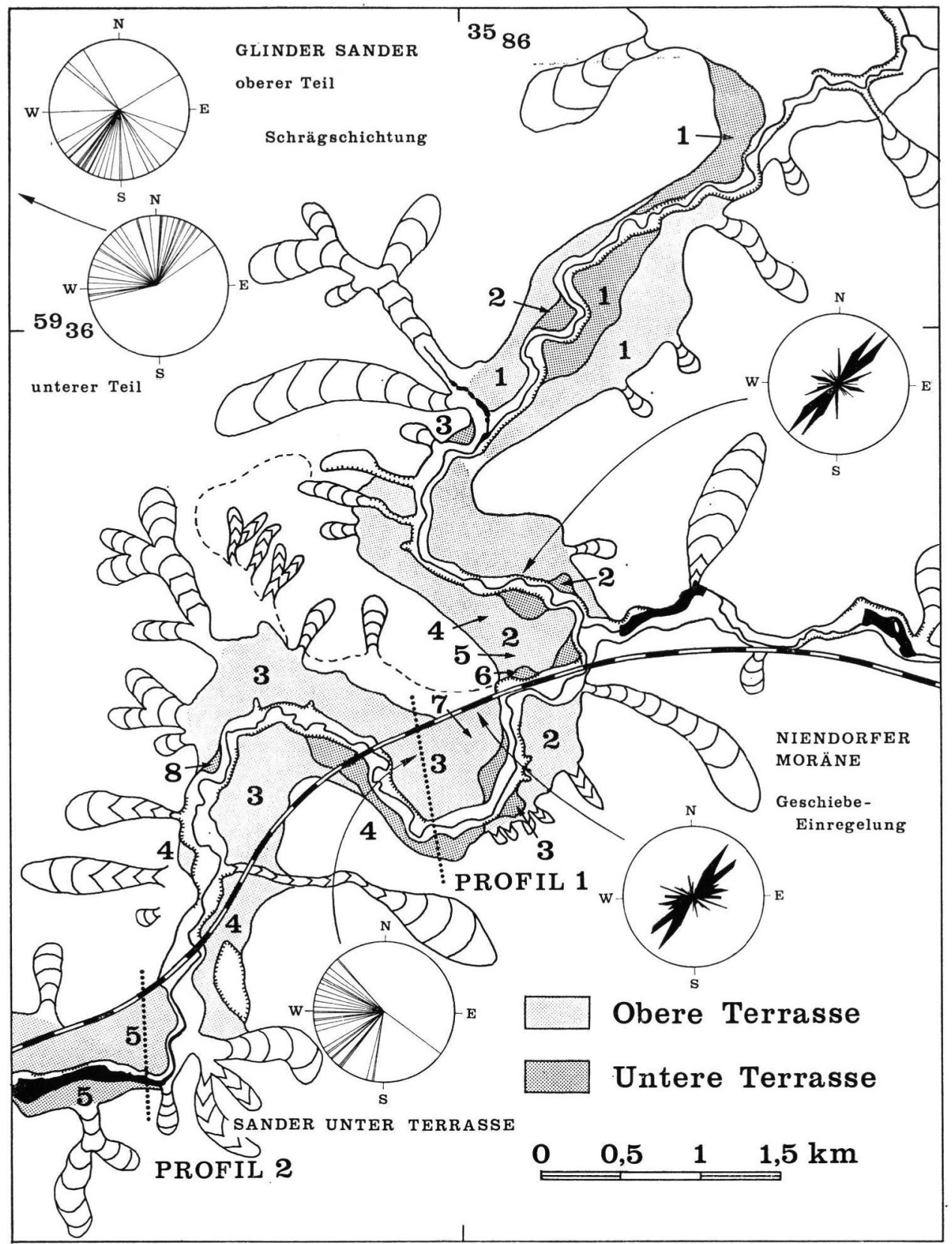

Abb. 4: Morphologische Karte des oberen Billetales. 


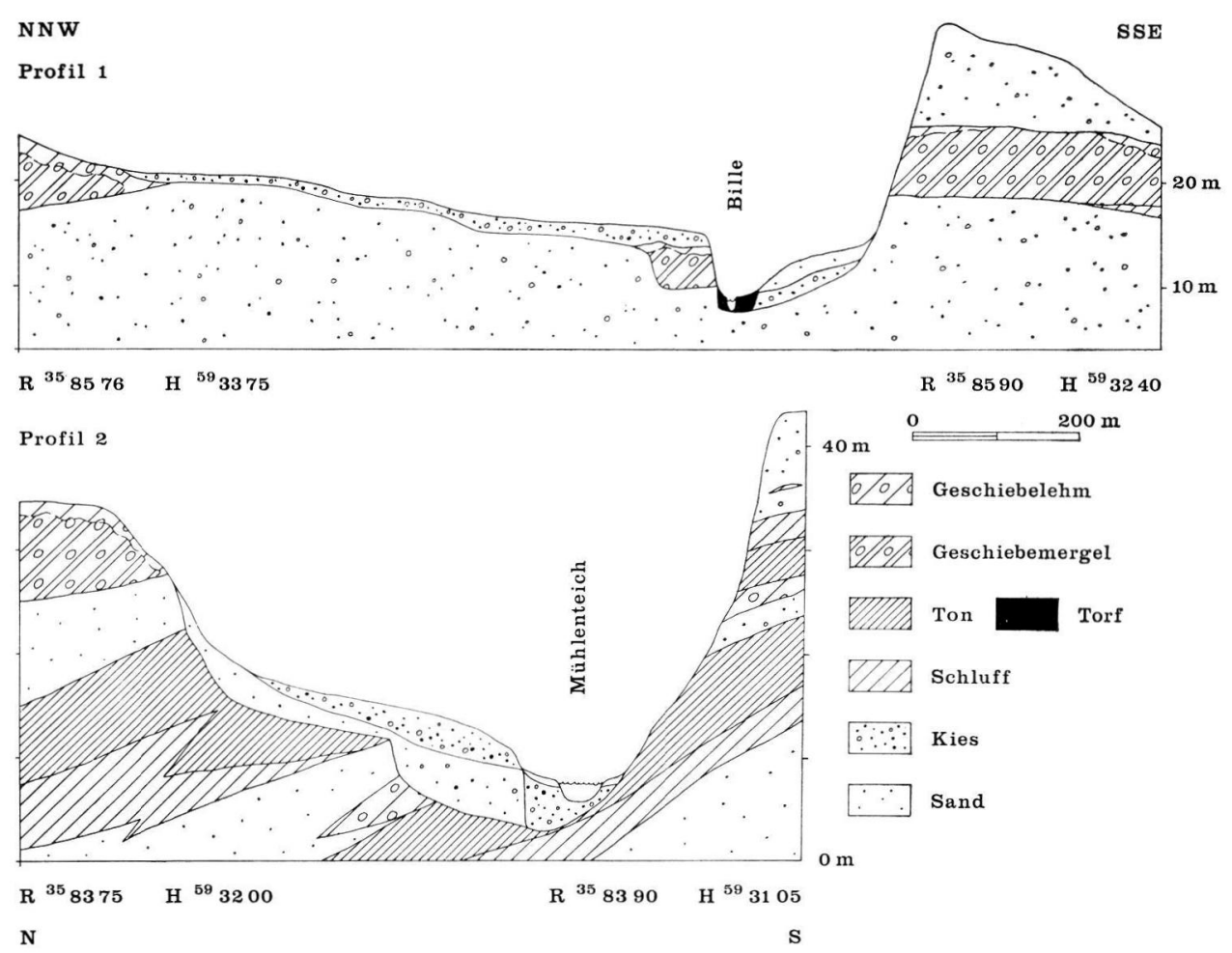

Abb. 5: Querprofile durch das Billetal am Krabbenkamp (Profil 1) und bei Reinbek (Profil 2).

jedoch beachtet werden, daß die viel länger dem Periglazialklima ausgesetzten Formen des oberen Terrassenniveaus zwangsläufig "verwaschener" erscheinen müssen als das „klare“, eng begrenzte Niveau der unteren Terrasse.

Die Terrassenkörper der oberen Terrasse sind aus Sanden, Kiesen und groben Schottern zusammengesetzt. Die Abbildungen 6 und 7 vermitteln einen Eindruck vom Terrassenmaterial, das sich von Schmelzwassersanden deutlich unterscheidet. Genauere Aussagen lassen die an insgesamt acht Proben durchgeführten Korngrößenanalysen zu (Abb. 8). Im Durchschnitt liegt der Skelettanteil $(>2 \mathrm{~mm}$ ) bei gut $30 \%$ und steigt im Extremfall sogar auf 56,97\% an. Aus diesem Grunde ist es nicht angebracht, die Terrasse weiterhin nach PfefFerle (1935) als "Tal s a n d terrasse“ zu bezeichnen.

Wie die Feinkiesanalyse (Abb. 9) beweist, unterscheidet sich die Terrasse in ihrer Geschiebezusammensetzung kaum von den liegenden Moränen- und Sandersedimenten. Das spricht für eine starke Aufarbeitung und Umlagerung von Lokalmaterial. Besonders das grobe Material dürfte hier in relativer Gletscherferne (am Krabbenkamp: mindestens $5 \mathrm{~km}$ ) fluviatil nicht weit transportiert worden sein. Wahrscheinlich handelt es sich um aufgearbeitetes Fließerdematerial von den Talhängen. Die glazifluviale Aufarbeitung zeigt sich in schräggeschichteten Partien und dachziegelartiger Lagerung der Gerölle. Die Terrassensedimente sind kalkfrei. 

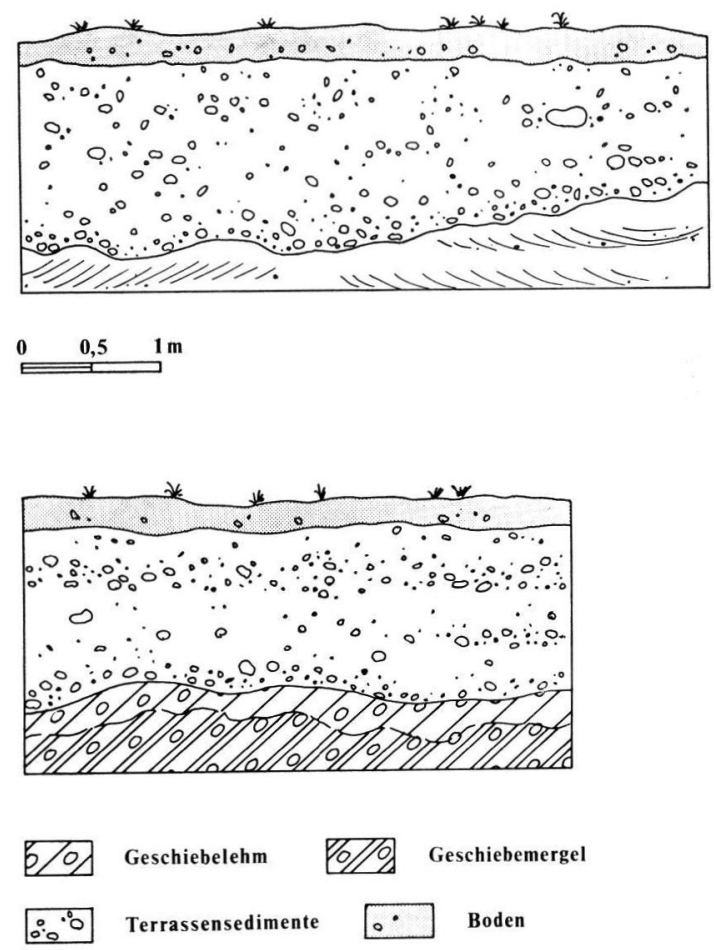

Abb. 6: Terrassenaufschlüsse in Baugruben am Krabbenkamp. Profile nicht überhöht. Oben = Terrassensedimente über Schmelzwassersanden $\mathrm{R} 35.857$ H 59.334. Unten = Terrassensedimente über Niendorfer Moräne R 35.858 H 59.331.

\section{Der Aufbau des Arbeitsgebietes}

Nach den Unterlagen des Archivs des Geologischen Landesamtes Hamburg wurden zwei Querprofile durch das Billetal konstruiert (Lage s. Abb. 4). Profil 1 liegt am Krabbenkamp. Das Profil zeigt folgenden Aufbau: Über grobkörnigen Schmelzwassersanden, die in mehreren Baugruben und im Einschnitt der Straßenunterführung zum Krabbenkamp aufgeschlossen waren, lagert in einer Höhe von etwa $17 \mathrm{~m}$ über $\mathrm{NN}$ eine graue, relativ tonige, kreidereiche Moräne, die etwa $5 \mathrm{~m}$ mächtig ist und südlich der Bille von etwa $10 \mathrm{~m}$ mächtigen Schmelzwassersanden überlagert wird. Auf Grund von Feinkieszählungen, Geschiebeeinregelungsmessungen und der stratigraphischen Position dieser Moräne ist anzunehmen, daß es sich um die Niendorfer Moräne (Grube 1967: 180f.) handelt. Die Niendorfer Moräne (in Schleswig-Holstein: Warthe-Moräne, vgl. LANGE, Menke \& Picard 1979) ist die mittlere Saale-Grundmoräne im norddeutschen Raum.

Im Bereich des Billetales ist diese Moräne fast überall erodiert worden (die heutige Bille fließt hier in einem Niveau von etwa NN $+9 \mathrm{~m}$ ). In unmittelbarer Nähe der Bille tritt sie jedoch noch einmal in einem knapp $100 \mathrm{~m}$ breiten Vorkommen auf. Sie wurde hier in mehreren Baugrund-Bohrungen angetroffen und war auch in einer Baugrube (Abb. 6 unten) aufgeschlossen. Anzeichen für eine Stauchung der Moräne fanden sich nicht.

Es wäre demnach denkbar, daß eine Urform des Billetales bereits durch die Gletscher des Niendorfer Vorstoßes angelegt worden ist. Die Streichrichtung des Billetales entspricht 


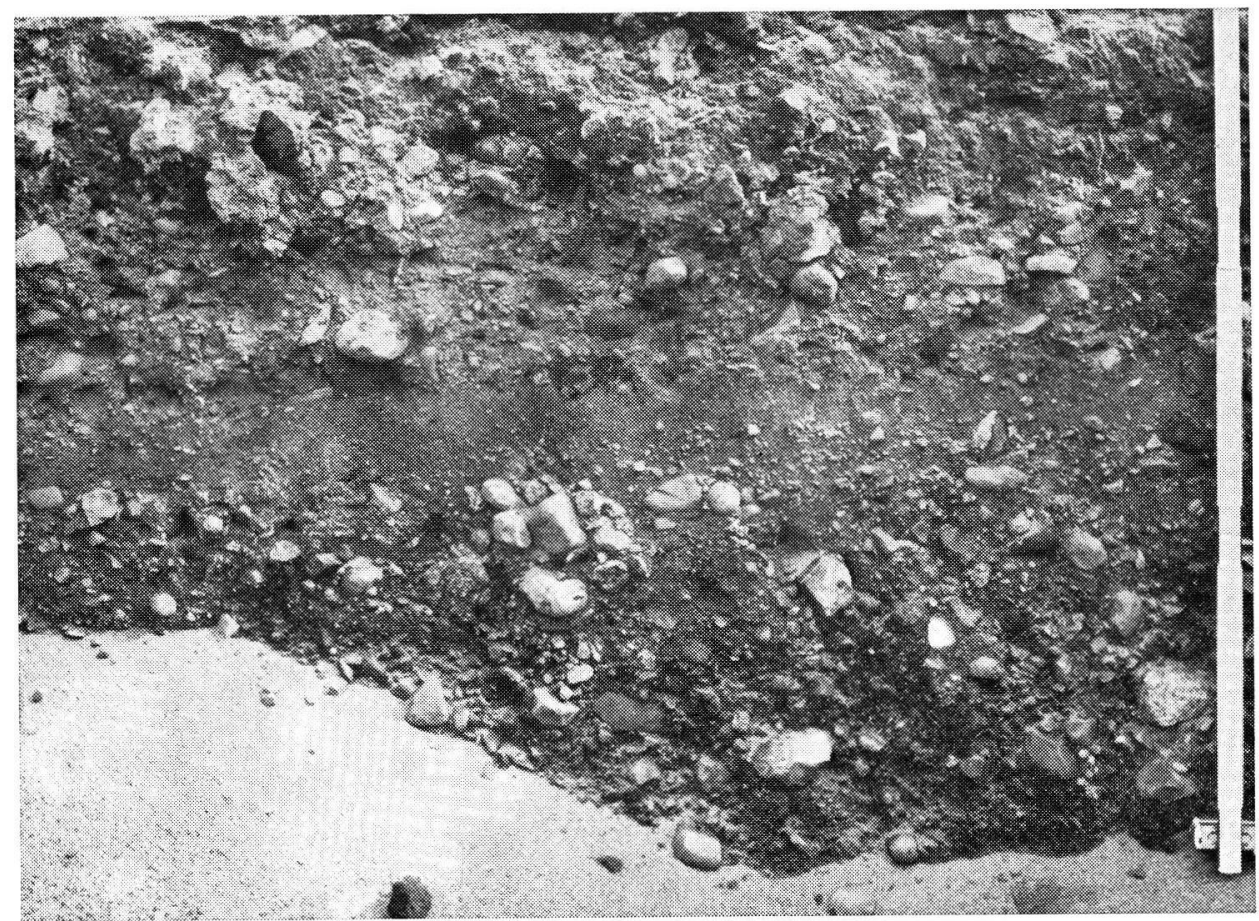

Abb. 7: Terrassensedimente der Bille am Krabbenkamp über Schmelzwassersanden. R 35.857 H 59.334 .

KORNGRÖSSENVERTEILUNG

Bille - Terrasse

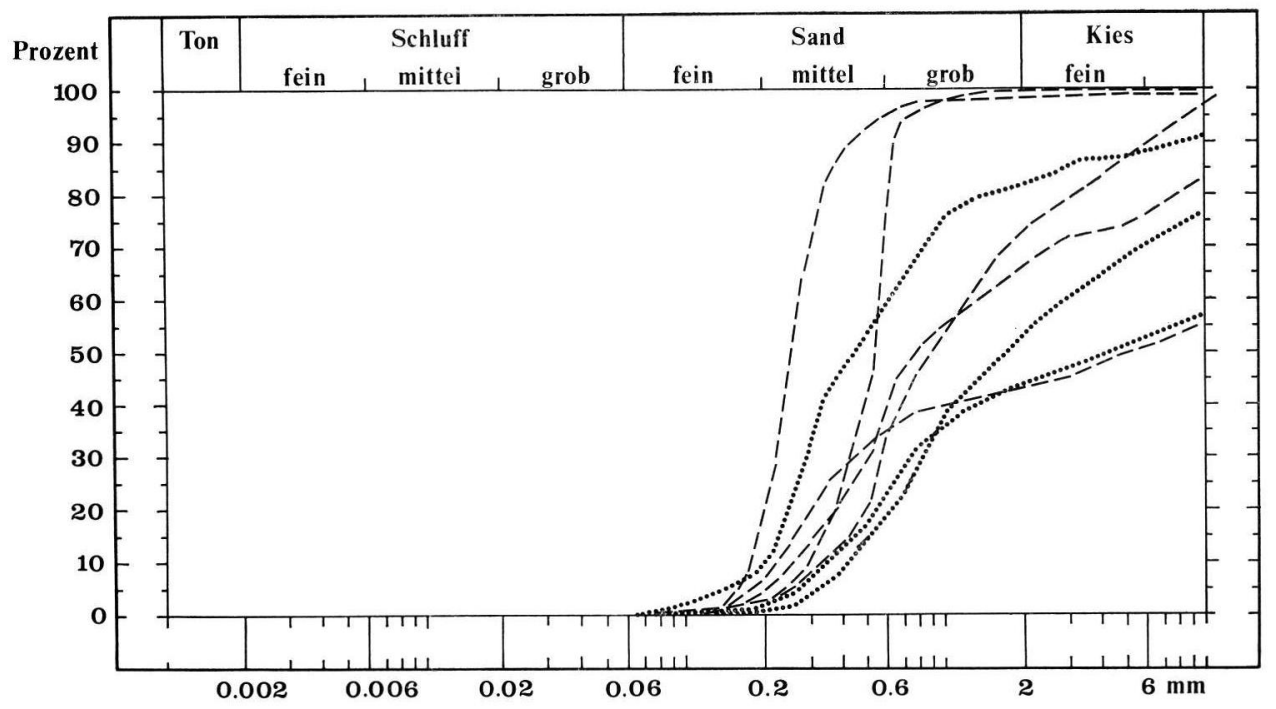

Abb. 8: Korngrößenverteilung der weichselzeitlichen Terrassensedimente.

Punktiert = Probe 11 bis 13 Baugrube am Krappenkamp, R 35.857 H 59.334 s. Abb. 1 Lokalität 5 . Gestrichelt $=$ Probe 17 bis 21 Baugrube am Krabbenkamp, R 35.858 H 59.331 s. Abb. 1 Lokalität 6 . 


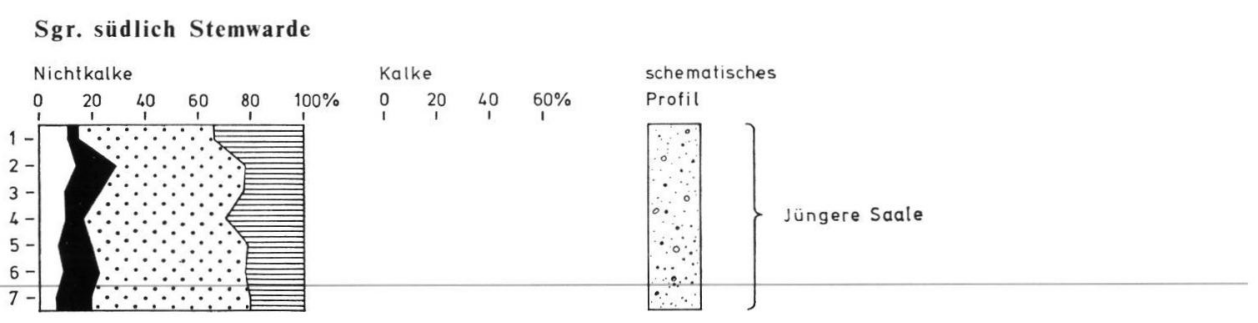

Kalksandsteinwerk Neu-Schönningstedt

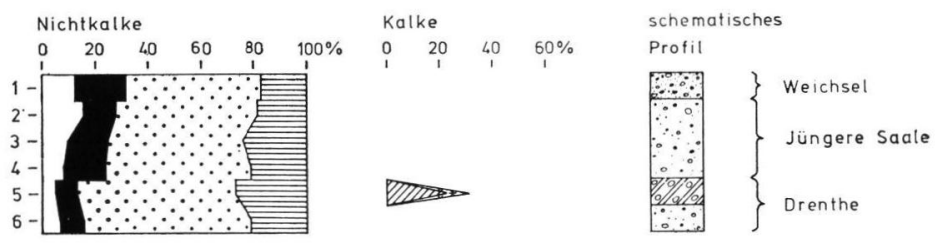

Abb. 9: Feinkiesanalysen aus dem Bereich des „Glinder Sanders“, Lage s. Abb. 1; Sgr. Neu-Schönningstedt R 35.820 H 59.375, Kalksandsteinwerk Neu-Schönningstedt R 35.835 H 59.358.

mit annähernd $40^{\circ}$ der gemessenen Eisbewegungsrichtung des Niendorfer Vorstoßes in diesem Gebiet. (Meßstellen: Sachsenwald und Krabbenkamp, vgl. Abb. 1, Lokalität 3 und 4). Eine Erhaltung der Talform bis in die heutige Zeit wäre trotz der nachfolgenden Überfahrung durch den Fuhlsbüttler Vorstoß nicht auszuschließen. So konnte EHLERS (1978 a: 66) nachweisen, daß das Kleckener Tal (Harburger Berge) während des Niendorfer Vorstoßes angelegt und vom Fuhlsbüttler Vorstoß überfahren worden ist, ohne daß die Talform zerstört wurde. Dagegen spricht jedoch, daß während des Fuhlsbüttler Vorstoßes (letzter Saale-Vorstoß im Hamburger Raum) der Glinder Sander aufgeschüttet worden ist (GRUBE 1967) - ein Vorgang, der unmöglich gewesen wäre, wenn zu dieser Zeit bereits die Möglichkeit des Abflusses durch das tiefer gelegene Billetal bestanden hätte.

Die Ausformung des Billetales zu seiner heutigen Gestalt erfolgte während der Weichsel-Eiszeit. Die Schmelzwässer des bei Witzhave liegenden Eisrandes haben dabei überwiegend erosiv gewirkt; lediglich die beschriebenen gut $1 \mathrm{~m}$ mächtigen Terrassenschotter liegen als dünne Sedimentdecke über den älteren Ablagerungen.

Nur während des Weichsel-Hochglazials wird dabei das damalige etwa $700 \mathrm{~m}$ breite Billetal in seiner ganzen Breite periodisch von Schmelzwässern durchflossen worden sein. Beim Abschmelzen des Inlandeises kam es - möglicherweise im Zusammenhang mit dem Auftauen des periglazialen Dauerfrostbodens - zur Einschneidung des heutigen, etwa $200 \mathrm{~m}$ breiten Billetales (Talaue), das auch jetzt noch zur Zeit der Schneeschmelze in voller Breite überflutet werden kann. 
Das zweite Profil bei Reinbek schneidet genau den Punkt, an dem Jaschke (1976: 79) sein stratigraphisches Profil aufgenommen hat. In diesem Bereich liegt eine ganze Anzahl tieferer Bohrungen vor. Der Untergrund ist hier stark gestaucht. Schichten des Tertiärs (Glimmerton und feinsandige Schluffe der Reinbek-Stufe des oberen Miozäns) sind von den Gletschern mit eiszeitlichen Ablagerungen verschuppt und bis in Höhen von über $\mathrm{NN}+20 \mathrm{~m}$ emporgepreßt worden.

In diese gestauchten Ablagerungen hat sich das Billetal eingeschnitten. Auf die Phase der Einschneidung folgte zunächst eine Ablagerung von relativ feinkörnigen Schmelzwassersanden, bevor im Weichsel-Hochglazial die Aufschotterung der Terrasse und schließlich im Spätglazial die erneute Einschneidung des heutigen Billetales erfolgte.

Mit Sicherheit findet sich an dieser Stelle im Billetal keine Moräne der Weichsel-Vereisung. Die von JaschKe (1976) beschriebenen Sedimente sind die groben Schotter der Bille-Terrasse, die zwar einer alpinen Fließ-Moräne ähneln, nicht aber einer Grundmoräne des Norddeutschen Tieflandes.

Im Folgenden soll der Aufbau der Umgebung des Billetales dargestellt werden. Während es auf der Grundmoränenebene des Sachsenwaldes kaum brauchbare Aufschlüsse gibt, bieten einige tiefere Gruben im westlich angrenzenden Bereich des Glinder Sanders Einblick in den Aufbau des Untergrundes.

In einer Sandgrube bei Neu-Schönningstedt (Abb. 1, Lokalität 1) waren über $20 \mathrm{~m}$ mächtige, meist schräggeschichtete Schmelzwassersande aufgeschlossen. Schrägschichtungsmessungen zeigten, daß es sich hierbei um zwei unterschiedliche Sanderschüttungen handelt. Der untere, feinkörnigere Teil der Sande ist nach NW geschüttet, der obere, gröbere Teil dagegen nach WSW. Grube (1967: 186) machte in den Gruben um Stemwarde ähnliche Beobachtungen. Er führt den Wechsel in der Fließrichtung darauf zurück, daß erst seit der Schüttung des oberen Teils des Sanders das Elbe-Urstromtal als Vorfluter zur Verfügung stand.

Die Sande sind völlig entkalkt und weisen erhebliche Anzeichen von Verwitterung auf. Dies zeigen auch Feinkiesanalysen (Abb. 9) aus der Sandgrube Neu-Schönningstedt (Abb. 1, Lokalität 1) und aus dem Aufschluß des Kalksandsteinwerkes Neu-Schönningstedt (Abb. 1, Lokalität 2). In dem Aufschluß des Kalksandsteinwerkes werden sie von einer in sich gestauchten Grundmoräne unterlagert, die auf Grund ihrer Feinkieszusammensetzung als Moräne des Drenthe-Vorstoßes angesprochen werden muß. Überlagert werden die Sande von einer Fließerde. In einer Mulde ist ein mehrere Dezimeter mächtiges Torf-Vorkommen aufgeschlossen, das vermutlich in die Eem-Warmzeit oder in ein Weichsel-Interstadial zu stellen ist.

Feinkieszusammensetzung und Schüttungsrichtung der Schmelzwassersande lassen vermuten, daß es sich tatsächlich - wie von GRUBE (1967: 186) behauptet - um Vorschüttsande des Fuhlsbüttler Vorstoßes handelt. Bei Dassendorf finden sich über der Niendorfer Moräne zwei jüngere Grundmoränen, von denen die ältere der Fuhlsbüttler Moräne entspricht. Die Jüngere ist gleichzusetzen mit der Warthe-Moräne MEYERs (1965) aus dem Lauenburger Raum (mdl. Mitt. Grube).

Korngrößenanalysen der Sander-Ablagerungen (Abb. 10) geben interessante Einblicke in die Sedimentationsbedingungen im Vorfeld des spätsaalezeitlichen Inlandeises. Selbst in den ausgesprochen kiesig wirkenden Partien geht der Kiesanteil der Proben nur in einem Teil knapp über 20\% hinaus; in allen Fällen überwiegen die Mittel- bis Grobsande. Während die kiesärmeren Proben eine sehr gleichmäßige Kornverteilung aufweisen, zeigt die Summenkurve der kiesreicheren Proben einen deutlichen Knick, das heißt, daß die Korngrößenverteilung zwei Maxima aufweist. Die Mehrgipfligkeit der Kurven ist darauf 
KORNGRÖSSENVERTEILUNG

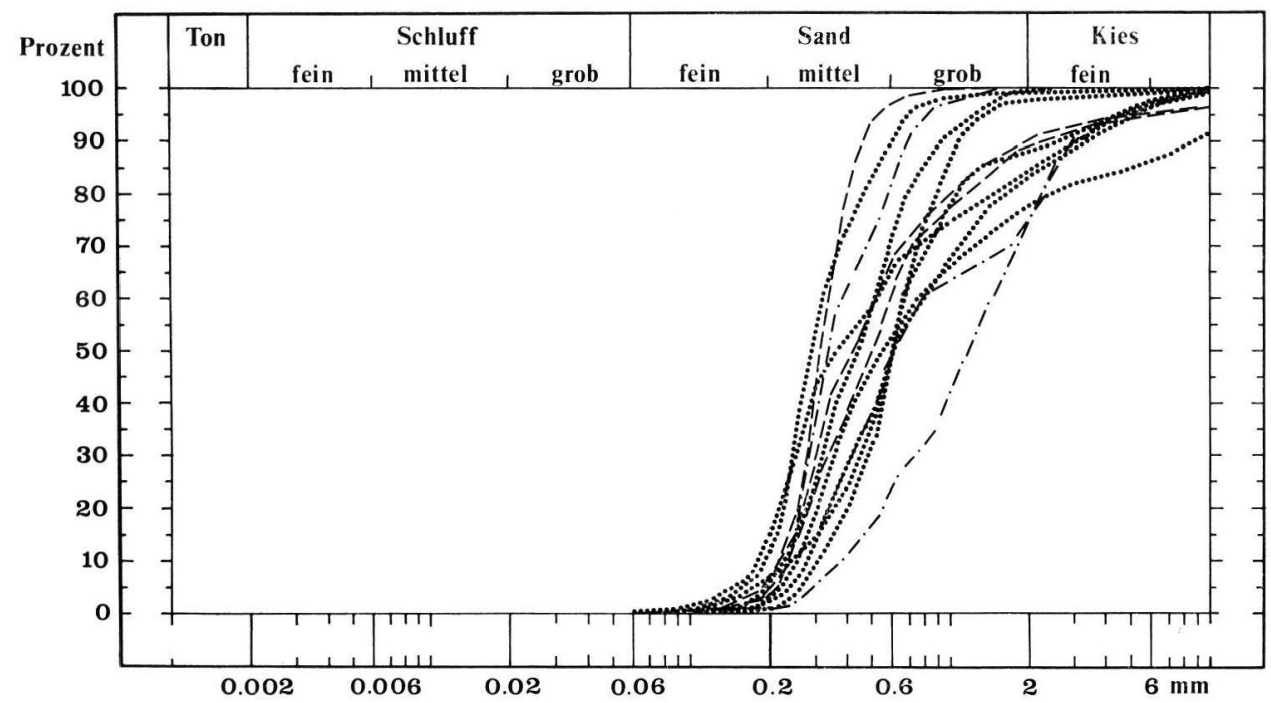

Abb. 10: Korngrößenverteilung der saalezeitlichen Sandersedimente.

Punktiert = Probe 1 bis 7 Oberer Teil des "Glinder Sanders“ R 35.857 H 59.334.

Gestrichelt $=$ Probe 8 bis 10 Unterer Teil des "Glinder Sanders" R 35.820 H 59.380.

Punktiert und gestrichelt $=$ Probe 14 bis 16 Sandersedimente unter der Billeterrasse am Krabbenkamp R 35.857 H 59.334 .

zurückzuführen, daß die Sedimente auf unterschiedliche Weise transportiert worden sind (vgl. Petтijohn 1975). Die groben Kiese wurden an der Sohle der Schmelzwasserflüsse gerollt, während sich die Masse der Sedimente durch Saltation fortbewegte und sich das Feinmaterial in Suspension befand. Offenbar ließ die hohe Fließgeschwindigkeit jedoch nicht $\mathrm{zu}, \mathrm{daß}$ suspendiertes Material in größerem Umfang hätte abgelagert werden können. Die Kurven weisen daher meist nur zwei Maxima auf.

Während die Umgebung des Billetales überwiegend aus Grundmoränen- oder Sander-Ebenen besteht, findet sich nordwestlich von Aumühle mit der Reliefeinheit Klingeberg-Hammelsberg eine endmoränenartige Form. Der Klingeberg streicht NW-SE und überragt mit seiner Höhe von 59,3 m NN die Umgebung um etwa $15 \mathrm{~m}$. Auf der Karte von DücKer (1958) ist hier eine Randlage der Saale-Vereisung eingetragen. In einem kleinen Aufschluß auf dem Klingeberg waren gestauchte Schmelzwassersande aufgeschlossen, die in einem Winkel von etwa $60^{\circ}$ nach E einfielen. Die Darstellung der Schichtflächen im Schmidt'schen Netz (vgl. Ehlers 1978 a: 17-23) zeigt, daß die Stauchung aus etwa $95^{\circ}$ erfolgt ist (Abb. 11). Es könnte sich damit um eine Stauchung durch den Fuhlsbüttler Vorstoß handeln, dessen Eis nachweislich im Harburger Raum aus dieser Richtung vorgestoßen ist (EHLERS 1975, 1978 a).

Von den drei im Hamburger Raum anstehenden Saale-Grundmoränen war im Bereich des Billetales selbst nur die Niendorfer Moräne an drei Stellen aufgeschlossen: in einer Baugrube und in einem Wegeinschnitt am Krabbenkamp sowie am Prallhang der Bille nördlich von Aumühle. An zwei Stellen konnten Geschiebe-Einregelungsmessungen 


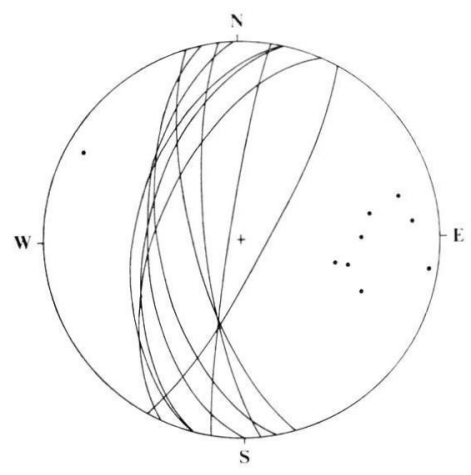

Abb. 11: Stauchungsmessungen am Klingeberg im Schmidt'schen Netz, untere Halbkugel R 35.849 H 59.351.

durchgeführt werden. Die Diagramme zeigen jeweils Maxima im Bereich von $35-45^{\circ}$. Die nordöstliche Eisvorstoßrichtung ist typisch für den Niendorfer Vorstoß im Harburger Raum (vgl. EHLers 1975, 1978 a).

Auch die Korngrößenanalysen zeigen eine starke Übereinstimmung mit den Werten, die Ehlers (1978 a: 6) für Niendorfer Moränen aus dem Süden Hamburgs ermittelt hat. Vergleichbar sind auch die Analysen, die Höfle (1979) für die Drenthe-2-Moräne (= Niendorfer Moräne) von Hemmoor angibt. Charakteristisch ist der sehr geringe Sortierungsgrad und der hohe Anteil von Schluff und Ton. Die Moränenproben-Analysen aus dem Billetal bestätigen den bereits im Gelände gewonnenen Eindruck, daß der Tongehalt der Niendorfer Moräne im Billetal offenbar relativ gering ist. Während in Hemmoor bis $15 \%$ Ton und in den Harburger Bergen 17-21\% Ton gemessen wurden, fanden sich im Billetal nur noch $12-14 \%$ Ton.

\section{Feinkiesanalysen}

Zur Absicherung der stratigraphischen Aussagen wurden im Untersuchungsgebiet an fünf Profilen Feinkiesanalysen vorgenommen (Abb. 12). Im Hamburger Raum ist es mit Hilfe von Feinkieszählungen der Fraktion 3-5 mm möglich, pleistozäne Schichtenfolgen altersmäßig einzustufen (EHLERs $1978 \mathrm{a}, \mathrm{b}$ ).

Am Prallhang der Bille im Sachsenwald war die Niendorfer Moräne in einem etwa $2 \mathrm{~m}$ hohen Anschnitt aufgeschlossen. Am Ort der Einregelungsmessung wurde auch eine Probe für eine Feinkieszählung entnommen. Das Ergebnis zeigt die für dieses Gebiet typischen Werte, bei denen vor allem der relativ hohe Gehalt an Schreibkreide auffällt. Daß er hier mit 16,4\% unter den Werten aus den anderen Profilen liegt, wird eine Folge der Verwitterung sein, die auf die Aufschlußwand eingewirkt hat.

Typisch für den Raum östlich von Hamburg ist der relativ geringe Flintgehalt der Niendorfer Moräne, der die Unterscheidung von anderen Ablagerungen der Saale-Eiszeit erschwert. Die Untersuchung einer Bohrung bei der Sternwarte in Bergedorf, die ein nahezu vollständiges Pleistozän-Profil mit drei Saale- und einer Elster-Moräne durchteuft hat, hat jedoch gezeigt, daß eine Unterscheidung hier dennoch möglich ist (mdl. Mitt. EHLERs). 


\section{Feinkiesanalyse Terrasse Krabbenkamp, Profil 1}

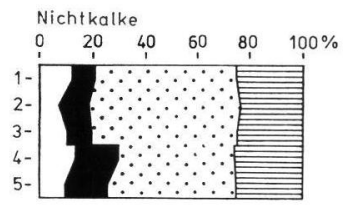

Kalke

$0 \quad 20 \quad 40 \quad 60 \%$

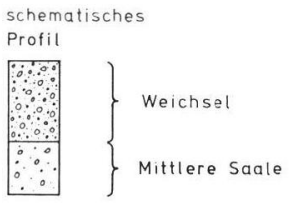

Feinkiesanalyse Terrasse Krabbenkamp, Profil 2 Nichtkalke

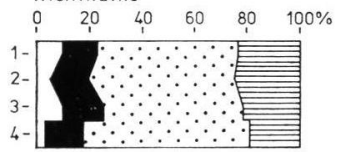

Kalke

$0 \quad 20 \quad 40 \quad 60 \%$

schematisches Profil

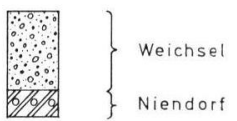

Feinkiesanalyse Lohbrügge, Billebogen
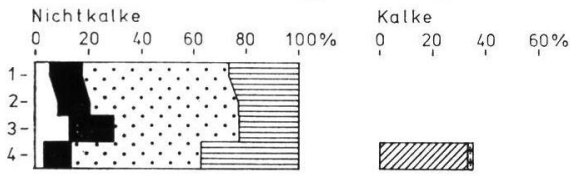

schematisches

Profil

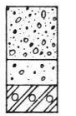

$\left\{\begin{array}{l}\text { Weichsel } \\ \{\text { Jüngere Saale } \\ \text { Niendorf }\end{array}\right.$

Unterführung Krabbenkamp
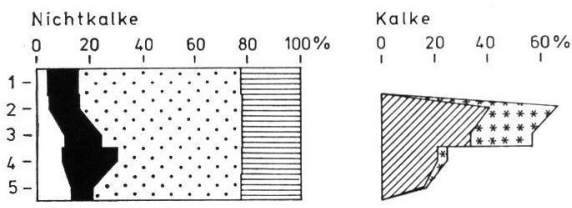

schematisches

Profil

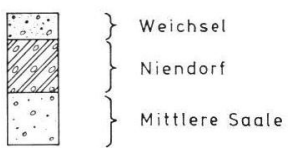

Billeufer, Sachsenwald
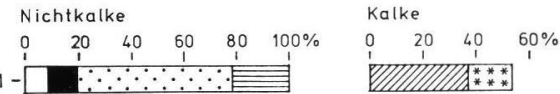

schematisches
Profil
Dolly Niendorf

Bille, Flußbett

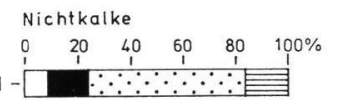

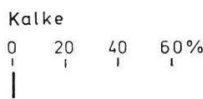

schematisches

Profil

$\because \because 9$ Holozan

Abb. 12: Feinkiesanalysen aus dem Bereich des Billetales, Lage s. Abb. 1

Unterführung Krabbenkamp

Billeufer, Sachsenwald

Bille, Flußbett

Terrasse Krabbenkamp, Profil 1

Terrasse Krabbenkamp, Profil 2

Lohbrügge, Billebogen
R 35.862 H 59.337

R 35.864 H 59.345

R 35.867 H 59.363

R 35.858 H 59.331

R 35.857 H 59.334

R 35.804 H 59.298 
Der Aufschluß an der Straßenunterführung am Krabbenkamp zeigt die Niendorfer Moräne über ihren Vorschüttsanden. Während in der Grundmoräne der Anteil der Kreidekalke zwischen 23,7 und 25,1 \% liegt, geht dieser in den Schmelzwassersanden auf 0,9 bis 3,7\% zurück. Auch der Gehalt an paläozoischen Kalken zeigt diesen Rückgang, der auf die stärkere Verwitterung der Sande zurückzuführen ist. Wo diese Schmelzwassersande nicht von einer Grundmoräne bedeckt sind und nicht unterhalb des Grundwasserspiegels liegen, sind sie viele Meter tief entkalkt (z. B. in der Sandgrube Neu-Schönningstedt).

Eine Baugrube am Krabbenkamp zeigte die Schmelzwasserschotter der Bille-Terrasse über älteren Schmelzwassersanden (vermutlich Vorschüttsanden des Niendorfer Vorstoßes), eine andere Baugrube ermöglichte Einblick in die Ablagerungen der Bille-Terrasse über der Grundmoräne des Niendorfer Vorstoßes. In beiden Fällen sind die Ablagerungen des weichselzeitlichen Billetales völlig entkalkt und zeigen auch sonst deutliche Zeichen der Verwitterung. Vor allem die dunklen Glimmer sind stärker angegriffen. Die Feinkieszusammensetzung ähnelt stark der der weichselzeitlichen Kiese des Elbe-Urstromtales (Ehlers 1980).

Eine Bohrung im Billebogen in Hamburg-Bergedorf nördlich des S-Bahnhofes Bergedorf erbrachte folgendes Profil (Lage vgl. Abb. 1, Lokalität 8):

$$
\begin{aligned}
& \text { 0-2,20 m Mittelsand, feinsandig, schwach grobsandig } \\
& -4,20 \mathrm{~m} \text { Fein- bis Mittelkies, grobsandig } \\
& -7,00 \mathrm{~m} \text { Geschiebemergel, tonig, grau. }
\end{aligned}
$$

Die Bohrung steht im Billetal in einer Höhe von $\mathrm{NN}+5,90 \mathrm{~m}$.

Die Feinkiesanalyse zeigt, daß auch hier unter den typischen Sanden des weichselzeitlichen Billetales die nahezu unverwitterte kreidereiche Grundmoräne des Niendorfer Vorstoßes ansteht. Hier erreicht der Kreidegehalt mit 36,6\% noch höhere Werte als im Bereich des Krabbenkamps und im Sachsenwald (Abb.9), wo maximal 25,1\% erreicht werden.

Die Feinkiesanalysen haben die Vermutungen über den geologischen Bau des Untersuchungsgebietes bestätigt. Bei den untersuchten Moränenproben handelt es sich jeweils um Material der Niendorfer Moräne. Hinweise auf weichselzeitliche Grundmoränen wurden nicht gefunden.

\section{Ergebnisse}

$\mathrm{Ob}$ das Billetal bereits während der Saale-Eiszeit vorgebildet worden ist, oder ob es sich um eine ausschließlich im Zuge der Weichsel-Vereisung entstandene Form handelt, läßt sich auf Grund der vorliegenden Untersuchung nicht eindeutig beantworten. Gewisse Hinweise sprechen jedoch dafür, daß das heutige Billetal auf eine während des Niendorfer Vorstoßes (mittlere Saale-Kaltzeit) gebildete Hohlform zurückgeht. Dagegen spricht jedoch, daß nach dem Niendorfer Vorstoß noch der hochliegende Glinder Sander neben dem Billetal aufgeschüttet worden ist.

Während des Hochglazials der Weichsel-Eiszeit reichte das nordische Inlandeis bis nach Witzhave am Nordrand des Untersuchungsgebietes. Seine Schmelzwässer erodierten das weite Billetal mit seinen großen Talmäandern und lagerten schließlich gut $1 \mathrm{~m}$ mächtige Terrassenschotter $a b$, die im Talverlauf in mehrere, aber wahrscheinlich annähernd zeitgleich gebildete Terrassenkörper zu untergliedern sind.

Als das Weichsel-Eis abschmolz, schnitten sich die Schmelzwässer stärker ein und formten das heutige, etwa $200 \mathrm{~m}$ breite innere Billetal, das heute nur während der Schneeschmelze noch in ganzer Breite vom Wasser durchflossen wird. 
Während des Holozäns wurden teils feinkörnige Sande, teils humose Sedimente abgelagert, in die sich die Bille - auf Grund der anthropogenen Entwaldung und des dadurch verstärkten Oberflächenabflusses - etwa einen Meter tief eingeschnitten hat.

Hinweise darauf, daß das Weichsel-Eis im Billetal bis an die Elbe vorgedrungen wäre, wurden während der Untersuchungen nicht gefunden. Nach Auswertung der Unterlagen des Geologischen Landesamtes Hamburg und nach Geländeuntersuchungen vermögen wir der Hypothese von JaschKe (1976) nicht zu folgen.

\section{Schriftenverzeichnis}

EhLERs, J. (1975): Neue Untersuchungen zur Entstehung der Harburger Berge. - Harburger Jb., 14: 7-49, 21 Abb., 1 Tab.; Harburg.

- (1978 a): Die quartäre Morphogenese der Harburger Berge und ihrer Umgebung. - Mitt. Geogr. Ges. Hamburg, 68: 181 S., 178 Abb., 47 Fot.; Hamburg.

- $(1978$ b): Feinkieszählungen nach der niederländischen Methode im Hamburger Raum. Der Geschiebesammler, 12 (2/3): 47-64, 7 Abb., 2 Fot.; Hamburg.

- (1980): Fine gravel analyses of drilling samples from the Hamburg area. - Meded. Rijks Geol. Dienst, 34 (7): 45-50, 6 figs.; Haarlem.

GRIPP, K. (1933): Geologie von Hamburg und seiner näheren und weiteren Umgebung. - 154 S., 35 Abb., 16 Taf.; Hamburg (Boysen in Komm.).

- (1964): Erdgeschichte von Schleswig-Holstein. - 411 S., 63 Abb., 57 Taf., 3 Kt.; Neumünster (Wachholtz).

Grube, F. (1967): Die Gliederung der Saale-(Riss-)Kaltzeit im Hamburger Raum. - Frühe Menschheit und Umwelt, 2: 168-195, 1 Tab., 8 Taf.; Köln/Graz.

- (1969): Zur Geologie der weichsel-eiszeitlichen Gletscherrandzone von Rahlstedt-Meiendorf. Ein Beitrag zur regionalen Geologie von Hamburg. - Abh. u. Verh. Naturwiss. Ver. Hamburg, N. F., 13: 141-194, 2 Abb., 1 Beil.; Hamburg.

HöFLE, H.-C. (1979): Klassifikation von Grundmoränen in Niedersachsen. - Ver. Naturwiss. Ver. Hamburg, N. F., 23: 81-92, 3 Abb., 1 Tab.; Hamburg.

Номсі, H. (1974): Jungpleistozäne Tunneltäler im Nordosten von Hamburg (Rahlstedt-Meiendorf). - Mitt. Geol. Paläont. Inst. Univ. Hamburg, 43: 99-126, 8 Abb.; Hamburg.

Illies, H. (1952): Die eiszeitliche Fluß- und Formengeschichte des Unterelbe-Gebietes. - Geol. Jb., 66: 525-558, 10 Abb.; Hannover.

JANZA, W. (1961): Bergedorf-Lohbrügge. Eine gewachsene Trabantenstadt Hamburgs. - Diss. Univ. Hamburg: 130 S., Anh.; Hamburg.

JASCHKE, D. (1976): Die weichselzeitliche Eisrandlage im Osten von Hamburg. - Eiszeitalter u. Gegenwart, 27: 75-81, 5 Abb., 1 Tab.; Ơhringen/Württ.

Lange, W., Menke, B. \& Picard, K.-E. (1979): Die Deutung glazigener Sedimente in SchleswigHolstein. - Verh. naturwiss. Ver. Hamburg, N. F., 23: 51-68, 2 Abb.; Hamburg.

MeYer, K.-D. (1965): Das Quartärprofil am Steilufer der Elbe bei Lauenburg. - Eiszeitalter u. Gegenwart, 16: 47-60, 3 Abb., 1 Taf.; OOhringen/Württ.

Olbricht, K. (1909): Grundlinien einer Landeskunde der Lüneburger Heide. - Forsch. dt. Landeskde. u. Volkskde., 18: 501-647, 4 Abb., 8 Taf.; Stuttgart.

Peтtijohn, E. J. (1975): Sedimentary Rocks. - 3rd ed., 628 pp., 347 figs., 86 tabs.; New York, Evanston, San Francisco and London (Harper \& Row).

Pfefferle, R. (1935): Zur Morphologie der Talsandterrassentäler in der Umgebung von Hamburg. - Diss. Univ. Hamburg: 77 S., 5 Taf.; Hamburg.

Stoller, J. (1914): Der jungdiluviale Lüneburger Eisvorstoß. - Jber. Niedersächs. Geol. Vereinig., 7: 214-230, $1 \mathrm{Kt}$; ; Hannover.

Uphoff, L. (1949): Von der Bille. - „Lichtwark“, Mitt.bl. des Lichtwark-Ausschusses in Bergedorf, 1, 3, 4; Hamburg-Bergedorf. 


\section{Verzeichnis der benutzten Karten und Luftbilder}

Topographische Karten:

1: 5000 Blatt Burgstall, Sachsenwaldau, Ohe (Kreis Stormarn), Aumühle, Wohltorf; Ausgabe 1958

Blatt Reinbek-Ost, Reinbek, Lohbrügge-Ost; 1959

Blatt Bergedorf; Ausgabe 1968

1: 25000 Blatt 2427 Glinde; Ausgabe 1974 und Erstausgabe von 1880

Blatt 2426 Wandsbek; Ausgabe 1956

Blatt 2328 Trittau; Ausgabe 1965

1: 50000 Blatt 2328 Trittau; Ausgabe 1968

Blatt 2528 Geesthacht; Ausgabe 1969

Blatt 2526 Hamburg-Wandsbek; Ausgabe 1965

Thematische Karten:

1: 20000 Gewässerkarte von Hamburg; Blatt 6818 Südost und 6832 Ost; Ausgabe 1976

1: 25000 Geologische Karte; Blatt 2427 Glinde; Berlin 1913

1 : 200000 Geologische Karte: Blatt Hamburg-Ost; Hannover 1977

$1: 300000$ Nordwestdeutschland; A. Bentz, 1951

1 : 500000 Geologische Karte von Schleswig-Holstein; A. Dücker, 1958

11 Luftbilder im Maßstab 1:12000 vom Lauf der Bille zwischen Witzhave und Reinbek (Objekt Willinghusen); Aufnahme 1977.

Manuskript eingegangen am 4. 5. 1981. 
